\title{
Intraprostatic fiducial markers: a potential application for ultrasound- guided radiotherapy in prostate cancer
}

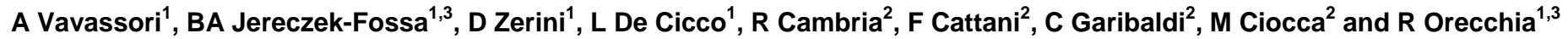 \\ ${ }^{1}$ Department of Radiation Oncology, European Institute of Oncology, 20141 Milan, Italy \\ ${ }^{2}$ Department of Medical Physics, European Institute of Oncology, 20141 Milan, Italy \\ ${ }^{3}$ University of Milan, European Institute of Oncology, 20141 Milan, Italy
}

\begin{abstract}
We report on a two-phase test performed to assess the ability of the ultrasound-based B-mode acquisition and targeting (BAT) transabdominal system to identify non-dedicated fiducial markers implanted into the prostate gland for subsequent image-guided radiotherapy.

Although further investigation is warranted in order to identify the optimal echogenic marker and to define its potential use for imageguided radiotherapy in prostate cancer patients, we demonstrate the feasibility of the BAT system for the visualization of non-ultrasounddedicated markers.
\end{abstract}

Published: $12 / 05 / 2009$

Received: 22/04/2009

ecancer 2009, 3:143 DOI: 10.3332/ecancer.2009.143

Copyright: (c) the authors; licensee ecancermedicalscience. This is an Open Access article distributed under the terms of the Creative Commons Attribution License (http://creativecommons.org/licenses/by/2.0), which permits unrestricted use, distribution, and reproduction in any medium, provided the original work is properly cited.

Competing Interests: The authors have declared that no competing interests exist.

Correspondence to A Vavassori. Email: andrea.vavassori@ieo.it 


\section{Introduction}

Dose escalation improves biochemical and clinical control in prostate cancer treated with external beam radiotherapy (EBRT), as demonstrated by several retrospective and prospective randomized studies [1-5]. Whenever a dose of $>70$ Gy is administered, the irradiated volume should be reduced in order to avoid excessive damage to the surrounding normal tissue. In clinical practice, such volume reduction has become feasible with the introduction of image-guided radiotherapy (IGRT). Various systems are routinely used in many departments for daily localization of the prostate during radiotherapy, i.e. implanted fiducial markers with $\mathrm{x}$-ray, onboard computed tomography (CT) or dedicated ultrasound (US) IGRT systems. Among these methods, the US system is probably the fastest. Several authors report that the fiducial marker-based IGRT is the most reliable, although it does not allow for soft tissue (prostate, seminal vesicles, rectum, urinary bladder) imaging [6].

In June 2005, the B-mode acquisition and targeting (BAT) transabdominal US System (Nomos, USA) was installed in our department. It is a computer-assisted cart-based system located in the treatment room enabling daily patient setup.

In a previous study [7], we evaluated the feasibility and accuracy of BAT-based prostate localization. Ten patients with localized prostatic adenocarcinoma were treated with threedimensional conformal radiotherapy (3D-CRT) to the dose of 72 Gy/30 fractions prescribed in the ICRU point (International Commission of Radiation Units). Daily US-based IGRT was compared to the electronic portal imaging (EPI) and CT-based alignment. We demonstrated that the BAT system ensures that the relative positions of the isocentre remain the same during treatment and as outlined in the treatment plan, even if the reliability of alignment is patient dependent. The average BATdetermined misalignments were small, confirming the prevalence of random errors in 3D-CRT. The limitations of the BAT system include inaccuracy of comparing images acquired by two different modalities (CT for planning and daily US images for setup), possible prostate displacement induced by US-probe pressure and operator dependence [8]. According to the literature, an average probe displacement of $12 \mathrm{~mm}$ results in an average prostate displacement of $3 \mathrm{~mm}[9,10]$. Prostate movement due to probe pressure is mostly in the anteroposterior (AP) direction, but systematic shifts have been observed also in the craniocaudal direction $[11,12]$.
An unpublished study on a series of 25 patients affected by localized prostate cancer treated at our department with hypofractionated 3D-CRT and daily BAT-based prostate localization showed a mean inter-patient systematic displacement of the prostate of $2.4 \mathrm{~mm}$ in the AP direction, probably related to the different US probe pressures used.

The purpose of this study was to test the potential role of two different commercially available fiducial markers in the USbased IGRT for prostate cancer. In the future, the introduction of fiducial markers for US-based IGRT might reduce both operator-dependent variations and potential influence of unfavourable patient anatomy, such as obesity or a small prostate located behind the pubic symphysis (patient dependence). Moreover, errors introduced during the learning curve for inexperienced US operators could be reduced.

\section{Materials and methods}

We tested the ability of the BAT system to visualize a set of standard gold fiducial markers (VisiCoil, RadioMed Corporation, USA; $0.75 \mathrm{~mm}$ in diameter and $10 \mathrm{~mm}$ in length) already used in our department for ExacTrac prostate cancer IGRT. In ExacTrac patients, the two VisiCoil fiducial markers are introduced into the prostate seven days before the simulation. For the purpose of the present study, we performed the BAT procedure in one patient enrolled in our ExacTrac $x$-ray hypofractionated protocol (2.7 Gy fraction; five fractions/week; 26 fractions, total dose of $70.2 \mathrm{~Gy}$ ).

The second part of our study involved the evaluation, in a lowrisk prostate cancer patient, selected for permanent seed implant brachytherapy, of the ability of the BAT system to visualize a different type of commercially available marker (CyberMark $^{\mathrm{TM}}$ Fiducial Marker, Civco, USA, $1 \mathrm{~mm}$ in diameter and $5 \mathrm{~mm}$ in length).

A dedicated informed consent was obtained. The implantation of the fiducial markers, near the left and right base positions, was performed using the same template-based brachytherapy technique.

Under epidural anaesthesia, the bladder was catheterized and filled with $200 \mathrm{cc}$ of saline solution. A well-trained operator performed a preliminary transabdominal US examination, using the BAT system, to check prostate visibility. 

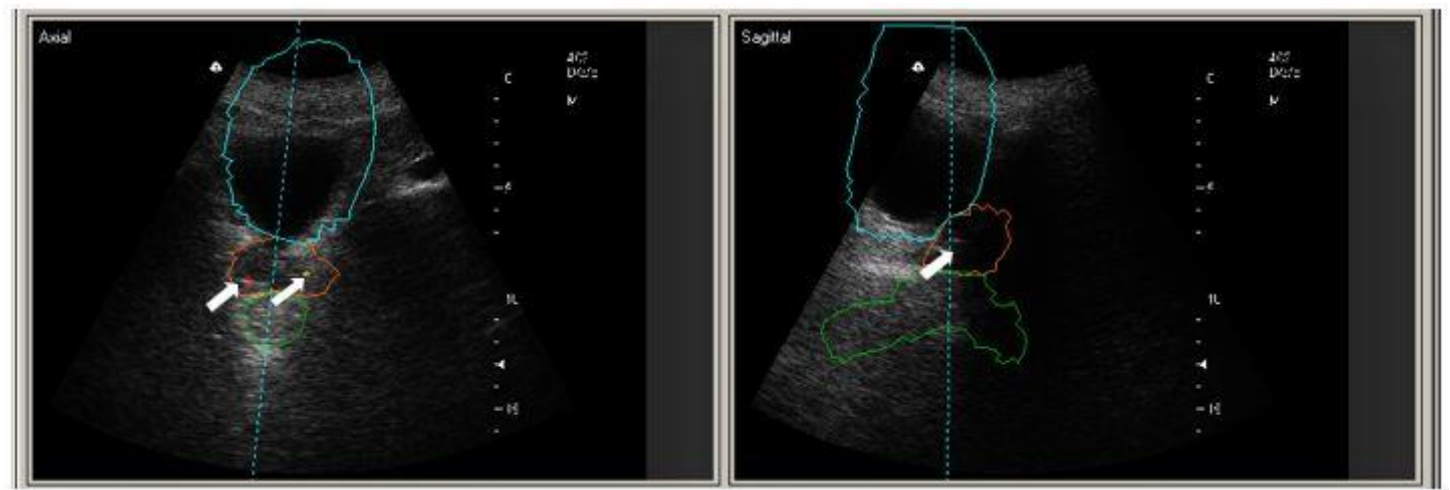

Figure 1: US-images and simulation-CT contours of the bladder, prostate and rectum in the IGRT patient with VisiCoil markers implanted (white arrows).
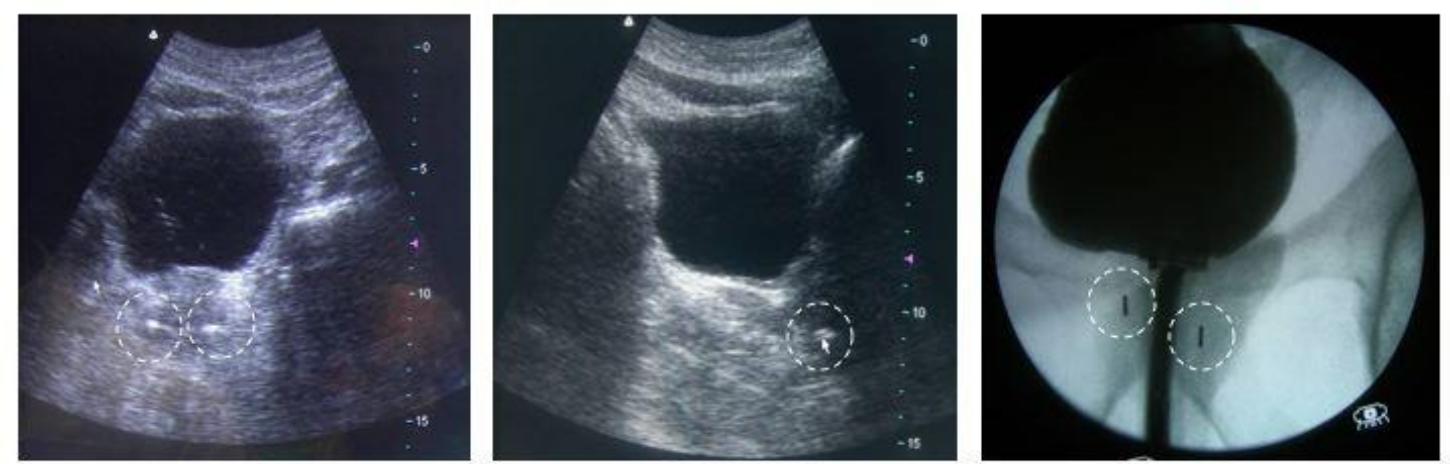

Figure 2: US and X-ray images of the CyberMark ${ }^{\mathrm{TM}}$ markers (dotted circles) in the brachytherapy patient.

Each marker was implanted via a transperineal percutaneous approach, using a dedicated preloaded needle under real-time transrectal US guidance.

At the end of the procedure, the same operator performed a new transabdominal US evaluation with the BAT system to visualize the markers inside the prostate and thereafter the planned brachytherapy procedure was started.

\section{Results}

These preliminary tests demonstrated the ability of the BAT system to visualize non-US-dedicated markers.

The alignment based on the fusion of the fiducial marker images coming from the simulation-CT and BAT procedure was feasible. The marker-based shift was equal to the soft-tissue imaging-based shift. The quality of the marker US image was satisfactory (Figure 1).

The time needed for implanting the markers in the brachytherapy patient was about 20 minutes and did not affect the accurate completion of the planned permanent seed implant (Figure 2).

The patient experienced no complications or discomfort after the two procedures.

\section{Discussion}

We have demonstrated the capacity of the BAT system to visualize different types of standard and commercially available non-US-dedicated fiducial markers.

The marker implantation is an invasive procedure with a potential for discomfort, possible bleeding, infection and 
prostate deformation [13]. However, if it proves applicable in clinical practice for US-based IGRT, as has been already demonstrated for cone-beam CT IGRT, it could reduce the subjectivity involved in interpreting the soft tissue image. In this way, the need for additional radiation doses from MV or $\mathrm{kV}$ radiography could be avoided. [14].

Moreover, the cost of US-based IGRT, even when fiducials are used, should be competitive with the CT or x-ray-based IGRT procedures.

\section{Conclusions}

Although the feasibility of using the BAT system with fiducial markers has been clearly demonstrated, new echogenic fiducials with different technical design should be tested to in order to facilitate the transabdominal US image evaluation [15].
Their construction material (gold, titanium, silver, carbon, etc.) should be carefully chosen to increase the proportion of US signal reflected depending on their different acoustic impedances. We believe that the results of this preliminary investigation warrant a more complete clinical study of the practical applicability of radiopaque echogenic markers in patients selected for daily BAT-based IGRT. Such a procedure might reduce inter-fraction and inter-observer discrepancies. Future studies, including 3-D-US technology, are warranted in order to increase the accuracy of US-based IGRT for prostate cancer.

\section{Conflict of interest}

The authors declare that they have no actual or potential conflicts of interest. 


\section{References}

1. Pollack A, Zagars GK, Starkschall G et al (2002) Prostate cancer radiation dose response: results of the MD Anderson phase III randomized trial Int J Radiat Oncol Biol Phys 53 1097-105 PMID: 12128107 doi: 10.1016/ S0360-3016(02)02829-8

2. Hanks GE, Hanlon AL, Epstein B and Horwitz EM (2002) Dose response in prostate cancer with 8-12 years' follow-up Int J Radiat Oncol Biol Phys 54 427-35 PMID: $\underline{12243818}$

3. Kupelian P, Kuban D, Thames $\mathrm{H}$ et al (2005) Improved biochemical relapse-free survival with increased external radiation doses in patients with localized prostate cancer: the combined experience of nine institutions in patients treated in 1994 and 1995 Int J Radiat Oncol Biol Phys 61 415-9 PMID: 15667961

4. Peeters ST, Heemsbergen WD, Koper PC et al (2006) Dose-response in radiotherapy for localized prostate cancer: results of the Dutch multicenter randomized phase III trial comparing 68 Gy of radiotherapy with 78 Gy J Clin Oncol 24 1990-6 PMID: 16648499 doi: 10.1200/ JCO.2005.05.2530

5. Kuban DA, Tucker SL, Dong L et al (2008) Long-term results of the MD Anderson randomized doseescalation trial for prostate cancer Int $\mathrm{J}$ Radiat Oncol Biol Phys 70 67-74 PMID: 17765406

6. Soete G, Verellen D and Storme G (2008) Image guided radiotherapy for prostate cancer Bull Cancer 95 374-80 PMID: 18390420

7. ICRU (1999) Report 62: Prescribing, Recording and Reporting Photon Beam Therapy (supplement to ICRU report 50) International Commission on Radiation Units and Measurements, Bethesda

8. Jereczek-Fossa BA, Cattani F, Garibaldi C et al (2007) Transabdominal ultrasonography, computed tomography and electronic portal imaging for 3- dimensional conformal radiotherapy for prostate cancer. Strahlenther Onkol 183 610-6 PMID: 17960336 doi: $10.1007 / \mathrm{s} 00066-007-1702-5$

9. Artignan $\mathrm{X}$, Smitsmans $\mathrm{MH}$, Lebesque JV, Jaffray DA, van Her $M$ and Bartelink $H$ (2004) Online ultrasound image guidance for radiotherapy of prostate cancer: impact of image acquisition on prostate displacement Int $\mathrm{J}$ Radiat Oncol Biol Phys 59 595-601 PMID: 15145181

10. McGahan J, Ryu J and Fogata M (2004) Ultrasound probe pressure as a source of error in prostate localization for external beam radiotherapy Int $\mathrm{J}$ Radiat Oncol Biol Phys 60 788-93 PMID: 15465195

11. Serago CF, Chungbin SJ, Buskirk SJ, Ezzel GA, Collie AC and Vora SA (2002) Initial experience with ultrasound localization for positioning prostate cancer patients for external beam radiotherapy Int J Radiat Oncol Biol Phys 53 1130-8 PMID: 12128112 doi: 10.1016/S0360-3016(02) 02826-2

12. Fung AY, Ayyangar KM, Djajaputra D, Nehru RM and Enke CA (2006) Ultrasound-based guidance of intensitymodulated radiation therapy Med Dosim 31 20-9 PMID: 16551526 doi: 10.1016/..meddos.2005.12.011

13. Nichol AM, Brock KK, Lockwood GA et al (2007) A magnetic resonance imaging study of prostate deformation relative to implanted gold fiducial markers Int J Radiat Oncol Biol Phys 67 48-56 PMID: 17084546

14. Johnston H, Hilts M, Beckham W and Berthelet E (2008) 3D ultrasound for prostate localization in radiation therapy: a comparison with implanted fiducial markers Med Phys 35 2403-13 PMID: 18649473 doi: 10.1118/1. $\underline{2818742}$

15. Davis BJ, Kinnick RR, Fatemi M, Lief EP, Robb RA and Greenleaf JF (2003) Measurement of the ultrasound backscatter signal from three seed types as a function of incidence angle: application to permanent prostate brachytherapy Int J Radiat Oncol Biol Phys 57 1174-82 PMID: 14575850 